\title{
The global prevalence of musculoskeletal disorders among operating room personnel: A systematic review and meta-analysis
}

\author{
Reza Tavakkol $^{\mathrm{a}}$, Esmaeil Kavi ${ }^{\mathrm{b}}$, Soheil Hassanipour ${ }^{\mathrm{c}, \mathrm{d}, * *}$, Hadiseh Rabiei $^{\mathrm{e}}$, \\ Mahdi Malakoutikhah ${ }^{\mathrm{f}, \mathrm{g}}$ \\ a Instructor of Operating room, Department of Operating room, School of Nursing, Shiraz University of Medical Sciences, Shiraz, Iran \\ ${ }^{\mathrm{b}}$ Instructor, Department of Nursing, School of Nursing, Larestan University of Medical Sciences, Larestan, Iran \\ ${ }^{c}$ Gastrointestinal and Liver Diseases Research Center, Guilan University of Medical Sciences, Rasht, Iran \\ ${ }^{\mathrm{d}}$ Caspian Digestive Disease Research Center, Guilan University of Medical Sciences, Rasht, Iran \\ ${ }^{\mathrm{e}}$ M.Sc. Student of Occupational Health Engineering, Department of Occupational Health Engineering, School of Public Health, Shahid Beheshti University of Medical \\ Sciences, Tehran, Iran \\ ${ }^{\mathrm{f}}$ Occupational Health Engineering, Department of Occupational Health Engineering, School of Public Health, Kashan University of Medical Sciences, Kashan, Iran \\ ${ }^{g}$ Student Research Committee, Shiraz University of Medical Sciences, Shiraz, Iran
}

\section{A R T I C L E I N F O}

\section{Keywords:}

Musculoskeletal symptom

Operating rooms

Meta-analysis

Systematic reviews

\begin{abstract}
A B S T R A C T
Objectives: Musculoskeletal disorders (MSDs) are one of the most important occupational problems. However, there has also been no worldwide review and meta-analysis of the prevalence of MSDs among operation room personnel. The present study aimed to investigate and estimate the prevalence of MSDs among operating room personnel using a systematic review and meta-analysis study.

Methods: This systematic review and meta-analysis were conducted in 2019. The researchers searched Medline/ PubMed, ProQuest, Scopus, ScienceDirect and Embase, and Google Scholar. Studies classified by categories of country and populations. In order to determine and control the quality assessment of articles, researchers use the Joanna Briggs Institute Critical Appraisal Checklist.

Results: A total of 111 articles from databases were found. Final screening included 12 into statistical analysis. Based on the random-effect model, the prevalence of MSDs in neck, shoulders, elbows, wrists \& hands, upper back, lower back, hip, knees, and ankles \& feet was 53.66\% (CI 95\%: 43.30-64.02), 55.63\% (CI 95\%: 43.90-67.36), 23.01\% (CI 95\%: 14.75-31.27), 38.79\% (CI 95\%: 21.95-55.62), 44.20\% (CI 95\%: 27.80-60.59), 61.48\% (CI 95\%: 44.33-78.64), 26.56\% (CI 95\%: 10.40-42.72), 43.43\% (CI 95\%: 23.80-63.06), and 57.06\% (CI 95\%: 48.25-65.87), respectively. Based on the results of meta-regression, there was no statistically significant relationship between prevalence of MSDs and mean of age, and year of study.

Conclusion: The study results revealed the high prevalence of MSDs, specifically in lower back among the operating room personnel. Considering the difficult conditions of working in the operating room, MSDs and particularly back pain are unpreventable.
\end{abstract}

\section{Introduction}

Musculoskeletal Disorders (MSDs) are amongst the main occupational health challenges in today's world and exist in numerous occupations. ${ }^{1-3}$ These disorders comprise one of the major issues in the field of health. ${ }^{4}$ According to the International Labor Organization's (ILO) report, out of the nearly 160 million work-related disorders occurring around the world annually, MSDs have been found to be the second most common occupational disease. ${ }^{5-7}$ MSDs have been defined as inflammatory and degenerative conditions that affect muscles, tendons, ligaments, joints, peripheral nerves, and supportive structures like intervertebral discs. ${ }^{8,9}$ These problems consist of a wide variety of disorders that differ in intensity and symptoms and can result in mild and moderate symptoms or chronic and disabling conditions. ${ }^{10,11}$

Statistics have indicated that MSDs accounted for a considerable amount of expenditures in healthcare departments, particularly healthcare personnel. They also exerted devastating effects on the personnel's health, quality of life, and job satisfaction and could lead to

\footnotetext{
* Corresponding author. Gastrointestinal and Liver Diseases Research Center, Guilan University of Medical Sciences, Razi Hospital, Sardar-Jangle Ave., P.O. Box: 41448-95655, Rasht, Iran.

E-mail address: Soheil.epid@gmail.com (S. Hassanipour).
} 
loss of workdays. ${ }^{12,13}$ Studies in the Europe demonstrated that almost 40 million workers (more than 30\%) suffered from these disorders, accounting for $0.5-2 \%$ of the European Union's Gross Domestic Product (GDP). ${ }^{2}$

Despite the high prevalence of MSDs in communities, healthcare personnel are faced more with these problems. ${ }^{14,15}$ Various investigations have shown that work-related disorders were one of the basic problems among healthcare personnel. Yet, the individuals who had direct contact with patients, specifically nurses and operating room personnel, were more involved with these disorders. ${ }^{16-18}$ The prevalence of MSDs has been reported to vary from $40 \%$ to $90 \%$ among nurses around the globe. ${ }^{19}$

According to the study by Andersen et al. and the Canadian Center for Occupational Health and Safety (CCOHS), various risk factors, including patient transfer, night shift stress, statistic stress (e.g., longterm standing, fixed body posture, and holding equipment such as surgical retractors), manual material handling (e.g., pulling, pushing, or lifting instruments, patients, or heavy equipment), and awkward posture alongside personal features such as age, sex, and obesity among operating room personnel could lead to the incidence of MSDs in the back, legs, and other body regions. ${ }^{20-24}$ Nonetheless, limited studies have been conducted on the prevalence of MSDs among operating room personnel. Evidence has indicated that operating room personnel were affected by musculoskeletal pain (58-90\%) after a year of clinical work. ${ }^{25}$ The limited studies performed on operating room personnel around the world have also revealed the high prevalence of these disorders. However, considering the debatable results obtained in this area, no comprehensive research has been conducted on the prevalence of MSDs among operating room personnel. Therefore, the present systematic review and meta-analysis aimed to explore the prevalence of MSDs among operating room personnel.

\section{Materials and methods}

The present systematic review and meta-analysis was conducted on the prevalence of MSDs among operating room personnel in 2019. The study was carried out on the basis of Preferred Reporting Items for Systematic Reviews and Meta-Analysis (PRISMA) checklist.

\subsection{Search strategy}

In the present study, researchers explored five international databases, namely Medline/PubMed, ProQuest, Scopus, ScienceDirect and Embase, in July 2019. In order for higher precision and investigation of the gray literature, Google Scholar was explored, as well. The selected keywords for international databases were classified into three categories as follows: 1-related to operating room personnel (operating room staff, operating room nurses, operation room technicians), 2- related to MSDs (musculoskeletal diseases, musculoskeletal pain, occupational diseases, occupational injuries, occupational health, cumulative trauma disorders, carpal tunnel syndrome, shoulder pain, neck pain, back pain, leg injuries, back injuries, neck injuries, arm injuries, hand injuries, muscle fatigue), and 3- related to prevalence (prevalence, frequency, incidence, epidemiology, posture, upper extremity, lower extremity).

The collected data were entered into the EndNote X8 software and the repeated articles were automatically omitted. It should be noted that the articles were separately evaluated by two researchers.

\subsection{Inclusion and exclusion criteria}

Generally, self-report questionnaires are used to estimate the prevalence of MSDs. The studies that had used questionnaires for investigating the prevalence of MSDs among operating room personnel were enrolled into the present research. However, the studies that had utilized MSDs risk assessment methods such as OWAS or REBA, those that were not conducted on operating room personnel, and those that had reported the risk factors of MSDs without expressing the prevalence of these disorders were excluded from the analysis.

\subsection{Evaluation of the articles quality}

In order to determine and control the articles quality, use was made of the Joanna Briggs Institute Critical Appraisal Checklist for Studies Reporting Prevalence Data. This instrument contains eight questions responded via three options; i.e., yes, no, and not applicable. The instrument aims at evaluation of the methodological quality of articles and determination of errors in studies, designs, and data analyses. In this checklist, the number of positive responses is computed and, accordingly, articles are categorized as low quality (scores 1 and 2 out of 9), moderate quality (scores 3-6 out of 9), and high quality (scores 7-9). In the present study, four articles had moderate quality and eight ones had high quality.

\subsection{Studies screening}

The primary search was carried out by two individuals (R.T and M.M). In addition, screening the studies, extraction of the results, and evaluation of the articles quality were separately done by two individuals (H.R and S.H). In case of disagreement between the two individuals, the team supervisor (E.K) made the final decision about the intended article.

\subsection{Statistical analysis}

Heterogeneity among the studies was assessed using Cochran's test (significance level $<0.1$ ) together with $\mathrm{I}^{2}$ statistic (significance level $>50 \%$ ). In case of heterogeneity, random effects model with inverse-variance approach was employed. In case of lack of heterogeneity, use was made of the fixed effects model. Moreover, meta-regression was used to assess the relationship between quantitative variables and the prevalence of MSDs. All analyses were performed using STATA software, version 13.

\section{Results}

\subsection{Description of searching for articles}

After searching all international databases, 111 articles were found. After deleting the repeated articles, 89 ones were entered into the title and abstract screening stage. Then, 18 articles were entered into the next stage where the full texts were explored and 10 articles were considered for the final analysis. In addition, two studies were selected after investigation of the articles' reference lists. After all, 12 studies were taken into consideration. In the screening stage, some articles were omitted due to irrelevance $(\mathrm{n}=61)$, irrelevant population $(\mathrm{n}=13)$, lack of access to the full text $(\mathrm{n}=3)$, and repeated findings $(\mathrm{n}=2)$ (Fig. 1).

\subsection{Description of the included studies}

All included studies were written in English. In addition, two studies were conducted in Iran, ${ }^{22,26}$ two in Saudi Arabia, ${ }^{27,28}$ two in the U.S., ${ }^{29,30}$ two in the Netherlands, ${ }^{31,32}$ one in Italy, ${ }^{33}$ one in Switzerland, ${ }^{25}$ and one in the Gaza Strip. ${ }^{34}$ Besides, the location was not specified in one study (Table 1).

The number of participants was mentioned in all included studies. In this regard, the smallest and largest number of participants was 32 and 463, respectively. Additionally, the participants' age was noted in 10 studies, indicating the mean (SD) of age of the participants to be 36.61 (3.82) years. Moreover, eight studies were conducted on both males and females, while gender was not specified in four studies. 

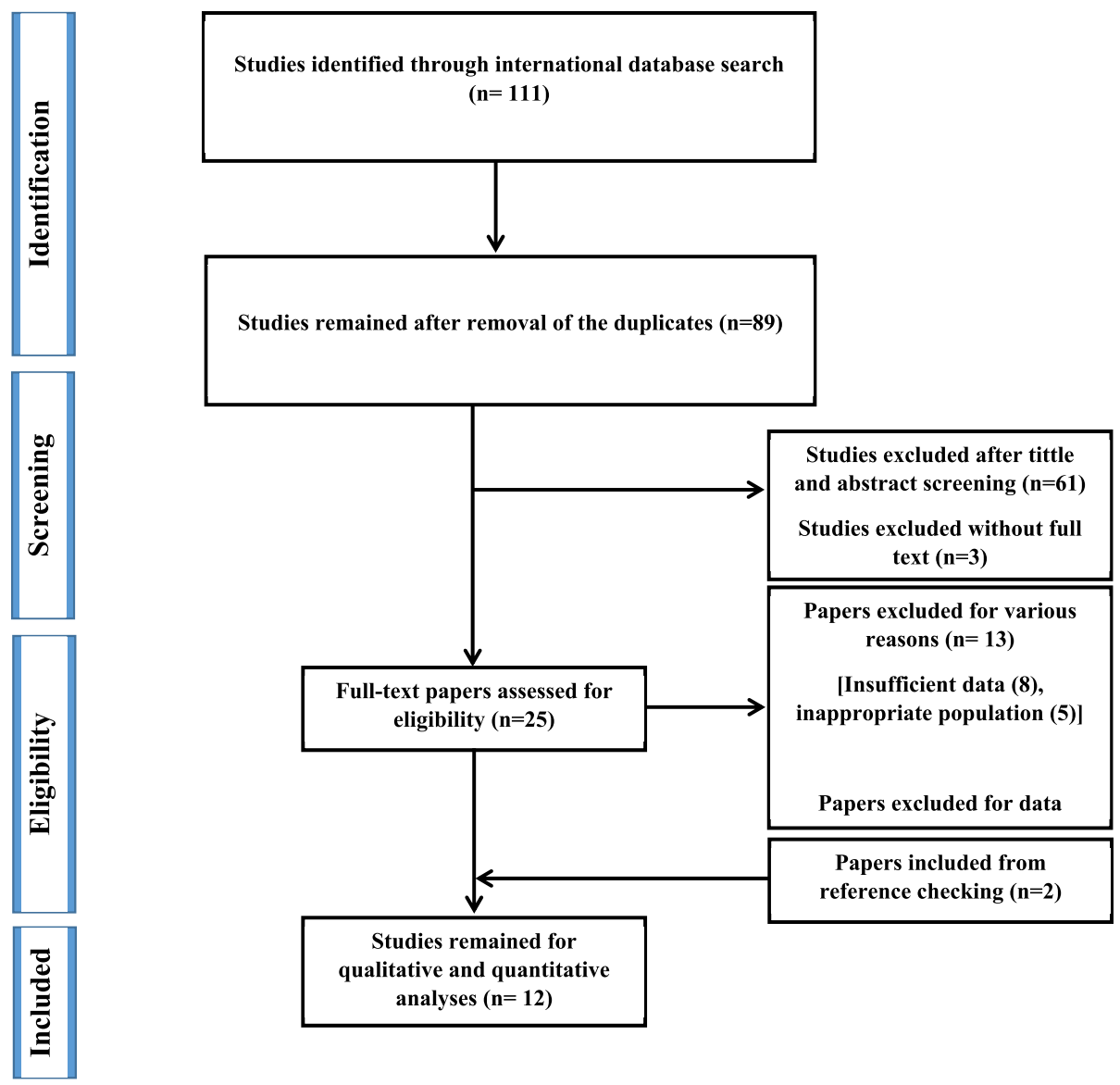

Fig. 1. Flowchart of the included studies in systematic review.

\subsection{Quality assessment of included studies}

The Joanna Briggs Institute checklist was used for quality assessment of articles. The results of the quality of studies showed that all studies had high quality.

\subsection{Results related to heterogeneity among the studies}

The results revealed great heterogeneity among the studies. Heterogeneity was assessed based on $\mathrm{Q}$ and $\mathrm{I}^{2}$ statistics, which revealed the following results: ankles \& feet $\left(\mathrm{Q}=24.60, \mathrm{df}=4, \mathrm{I}^{2}=83.70 \%\right.$, $\mathrm{p}<0.001)$, elbows $\left(\mathrm{Q}=26.17, \mathrm{df}=3, \mathrm{I}^{2}=88.5 \%\right.$, $\left.\mathrm{p}<0.001\right)$, hip $\left(\mathrm{Q}=81.86, \mathrm{df}=3, \mathrm{I}^{2}=96.30 \%, \mathrm{p}<0.001\right)$, knees $(\mathrm{Q}=187.83$, $\left.\mathrm{df}=4, \mathrm{I}^{2}=97.90 \%, \mathrm{p}<0.001\right)$, lower back $(\mathrm{Q}=1182.31, \mathrm{df}=11$, $\left.\mathrm{I}^{2}=99.10 \%, \mathrm{p}<0.001\right)$, neck $\left(\mathrm{Q}=8.47, \mathrm{df}=2, \mathrm{I}^{2}=76.40 \%\right.$, $\mathrm{p}<0.05)$, shoulders $\left(\mathrm{Q}=75.39, \mathrm{df}=4, \mathrm{I}^{2}=94.70 \%, \mathrm{p}<0.001\right)$, upper back $\left(\mathrm{Q}=22.02\right.$, df $\left.=2, \mathrm{I}^{2}=90.90 \%, \mathrm{p}<0.001\right)$, and wrists $\&$ hands $\left(\mathrm{Q}=144.84, \mathrm{df}=4, \mathrm{I}^{2}=97.20 \%, \mathrm{p}<0.001\right)$.

\subsection{The results of meta-analysis of the studies}

Considering the great heterogeneity among the results, the random effects model was used. The results of meta-analysis based on the prevalence in different body regions are as follows:

\subsection{Neck}

The prevalence of MSDs in the neck was reported in three studies carried out in Iran and the U.S. (Fig. 2). Based on the results of the random effects model, the prevalence of this disorder was $53.66 \%$

Table 1

Summery of the studies included in systematic review and meta-analysis.

\begin{tabular}{|c|c|c|c|c|c|c|}
\hline Order & Author, year & Country & Sample size & Population & Gender & Mean of age (SD) \\
\hline 1 & Choobineh, 2010 & Iran & 375 & Operating room nurses & Both & $31.54(8.46)$ \\
\hline 2 & Asghari, 2019 & Iran & 144 & Operating room nurses & Both & $34.6(6.6)$ \\
\hline 3 & Jeyakumar, 2018 & - & 250 & Operating room nurses & - & - \\
\hline 4 & Bin Homaid, 2016 & Saudi Arabia & 120 & Operation room staff & Both & $33.9(7.6)$ \\
\hline 5 & Marina Nützi, 2015 & Switzerland & 116 & Operating room nurses & Both & $39.94(11.90)$ \\
\hline 6 & Keriri, 2013 & Saudi Arabia & 126 & Operating room nurses & Both & $34.03(8.02)$ \\
\hline 7 & Yousef Aljeesh, 2011 & Gaza Government & 143 & Operating room nurses & Both & $33.7(9.6)$ \\
\hline 8 & Moscato, 2010 & Italy & 185 & Operating room nurses & Both & $36.08(7.08)$ \\
\hline 9 & Sheikhzadeh, 2009 & U.S.A & 32 & Operating room nurses & - & $43.9(9.1)$ \\
\hline 10 & Meijsen, 2007 & Netherlands & 463 & Perioperative personnel & - & - \\
\hline 11 & Ellen Bos, 2007 & Netherlands & 381 & Operating room nurses & Both & $40^{10}$ \\
\hline 12 & Diaz, 2001 & U.S.A & 181 & Operating room nurses & - & $38.41(7.48)$ \\
\hline
\end{tabular}


$\begin{array}{lll}\text { Author_Year } \quad \text { Country } & \text { ES }(95 \% \mathrm{Cl})\end{array}$

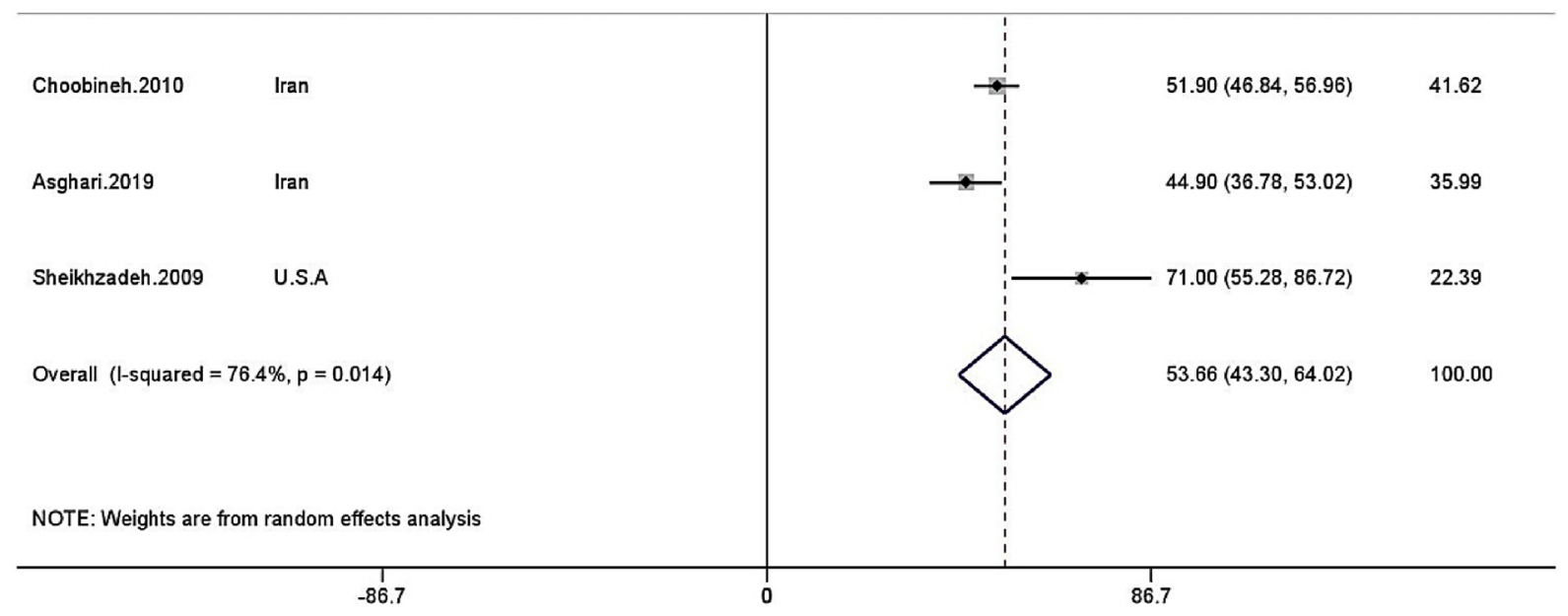

Fig. 2. Prevalence of musculoskeletal disorders in neck amongst studies included.

(Confidence Interval (CI 95\%): 43.30-64.02).

\subsection{Shoulders}

According to Fig. 3, the prevalence of MSDs in shoulders was mentioned in five studies performed in Iran, the U.S., and the Netherlands. Based on the results of the random effects model, the total prevalence of this disorder was 55.63\% (CI 95\%: 43.90-67.36).

\subsection{Elbows}

Based on the results, four studies conducted in Iran, the U.S., and the Netherlands showed the prevalence of MSDs in elbows. The results of the random effects model revealed the prevalence of this disorder to be $23.01 \%$ (CI 95\%: 14.75-31.27) (Fig. 4).

\subsection{Wrists and hands}

A total of five studies conducted in Iran, the U.S., Switzerland, and the Netherlands revealed the prevalence of MSDs in wrists \& hands. According to the results of the random effects model, the prevalence of this disorder was found to be $38.79 \%$ (CI 95\%: 21.95-55.62) (Fig. 5).

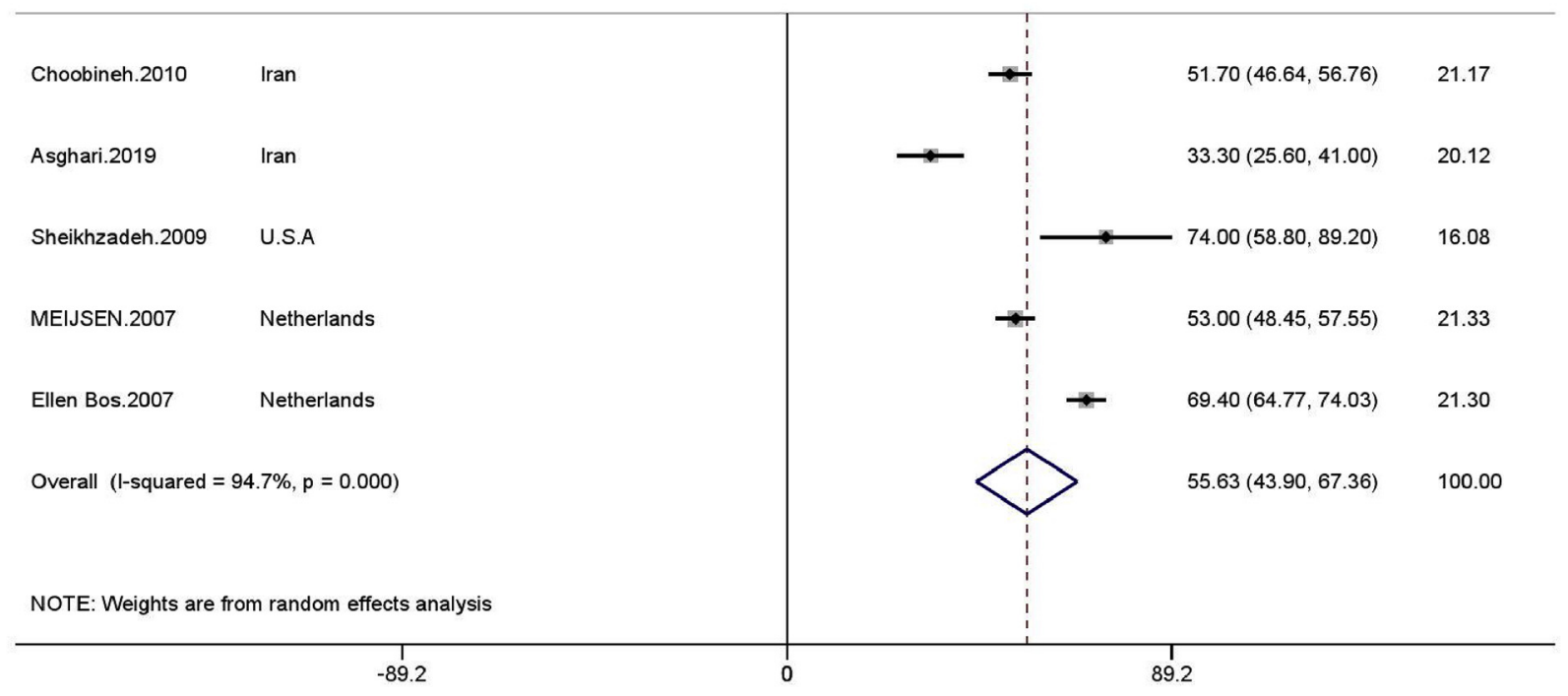

Fig. 3. Prevalence of musculoskeletal disorders in shoulders amongst studies included. 


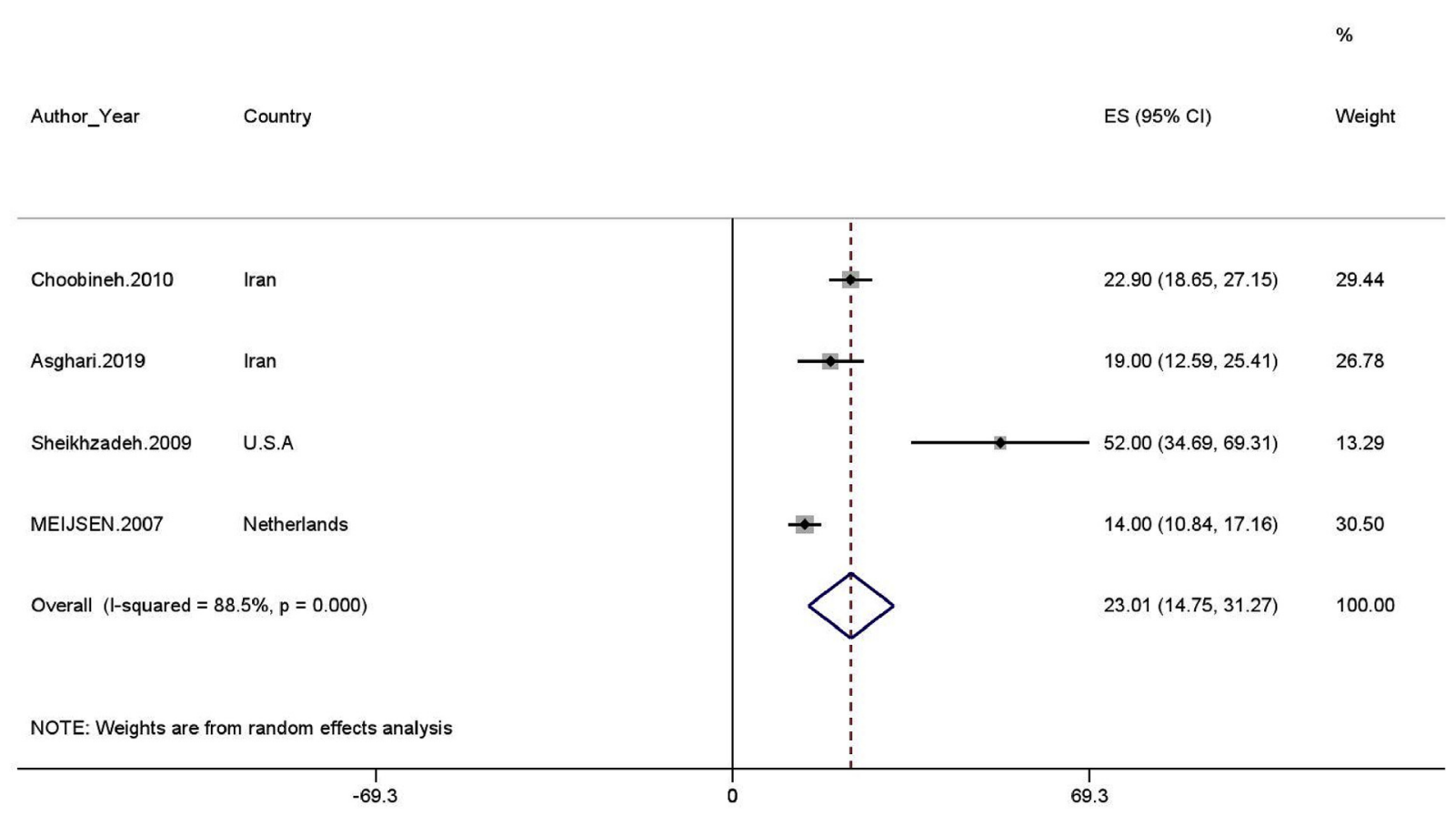

Fig. 4. Prevalence of musculoskeletal disorders in elbows amongst studies included.

\subsection{Upper back}

The prevalence of upper back MSDs has been depicted in Fig. 6. Accordingly, the prevalence of upper back MSDs was reported in three studies carried out in Iran and the U.S. The results of the random effects model demonstrated that the prevalence of this disorder was $44.20 \%$ (CI 95\%: 27.80-60.59).

\subsection{Lower back}

According to Fig. 7, the prevalence of lower back MSDs was mentioned in 12 studies. The results of the random effects model showed that the prevalence of this disorder was $61.48 \%$ (CI 95\%: 44.33-78.64).

\subsection{Hip}

The prevalence of hip MSDs has been presented in Fig. 8. This was assessed in four studies performed in Iran, the U.S., and Switzerland. Based on the results of the random effects model, the prevalence of this disorder was $26.56 \%$ (CI 95\%: 10.40-42.72).

\subsection{Knees}

The prevalence of MSDs in knees was reported in five studies carried

$\%$

Author_Year Country

ES $(95 \% \mathrm{Cl})$

Weight

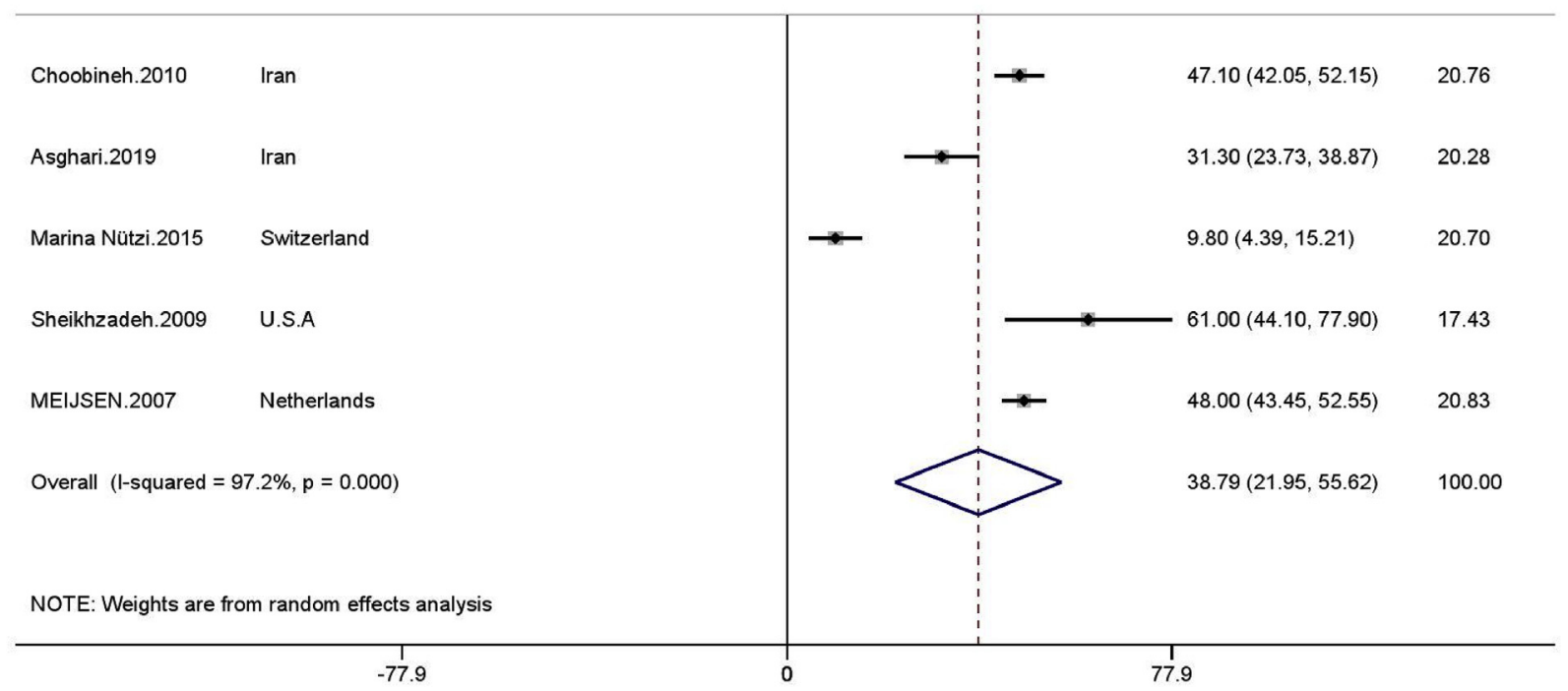

Fig. 5. Prevalence of musculoskeletal disorders in wrist \& hand amongst studies included. 


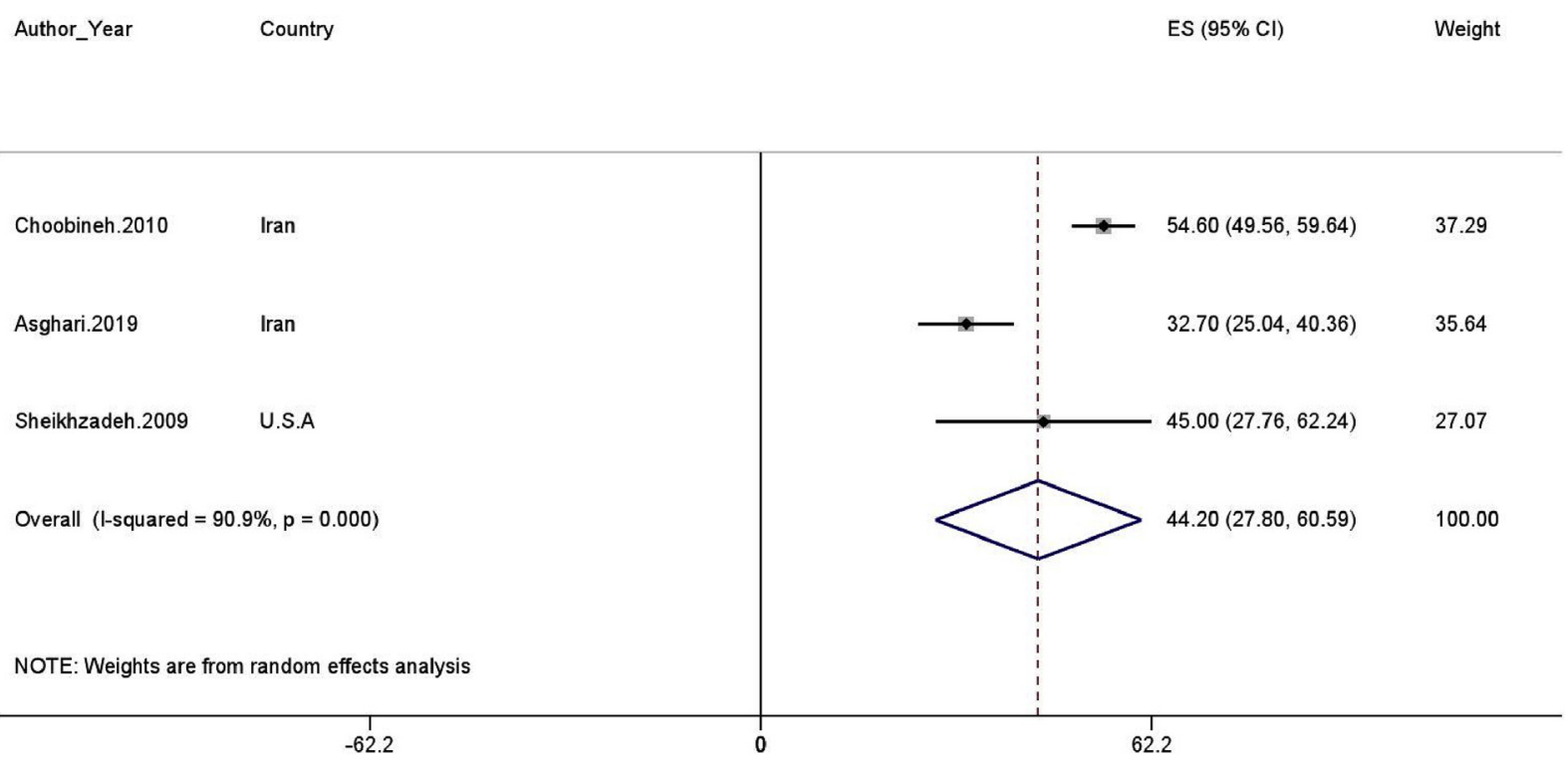

Fig. 6. Prevalence of musculoskeletal disorders in upper back amongst studies included.

out in Iran, the U.S., Switzerland, and the Netherlands. Based on the results of the random effects model, the prevalence of this disorder was 43.43\% (CI 95\%: 23.80-63.06) (Fig. 9).

\subsection{Ankles \& feet}

The prevalence of MSDs in ankles \& feet was reported in five studies conducted in Iran, the U.S., Switzerland, and Italy. The results of the random effects model showed that the prevalence of this disorder was
57.06\% (CI 95\%: 48.25-65.87) (Fig. 10).

\subsection{Meta-regression}

Meta-regression test was used to evaluate the impact of various factors on the prevalence of MSDs in different body regions. The results of investigation of the relationship between the year of study and the prevalence of MSDs indicated a change in the prevalence of these disorders in the recent years, but the change was not statistically

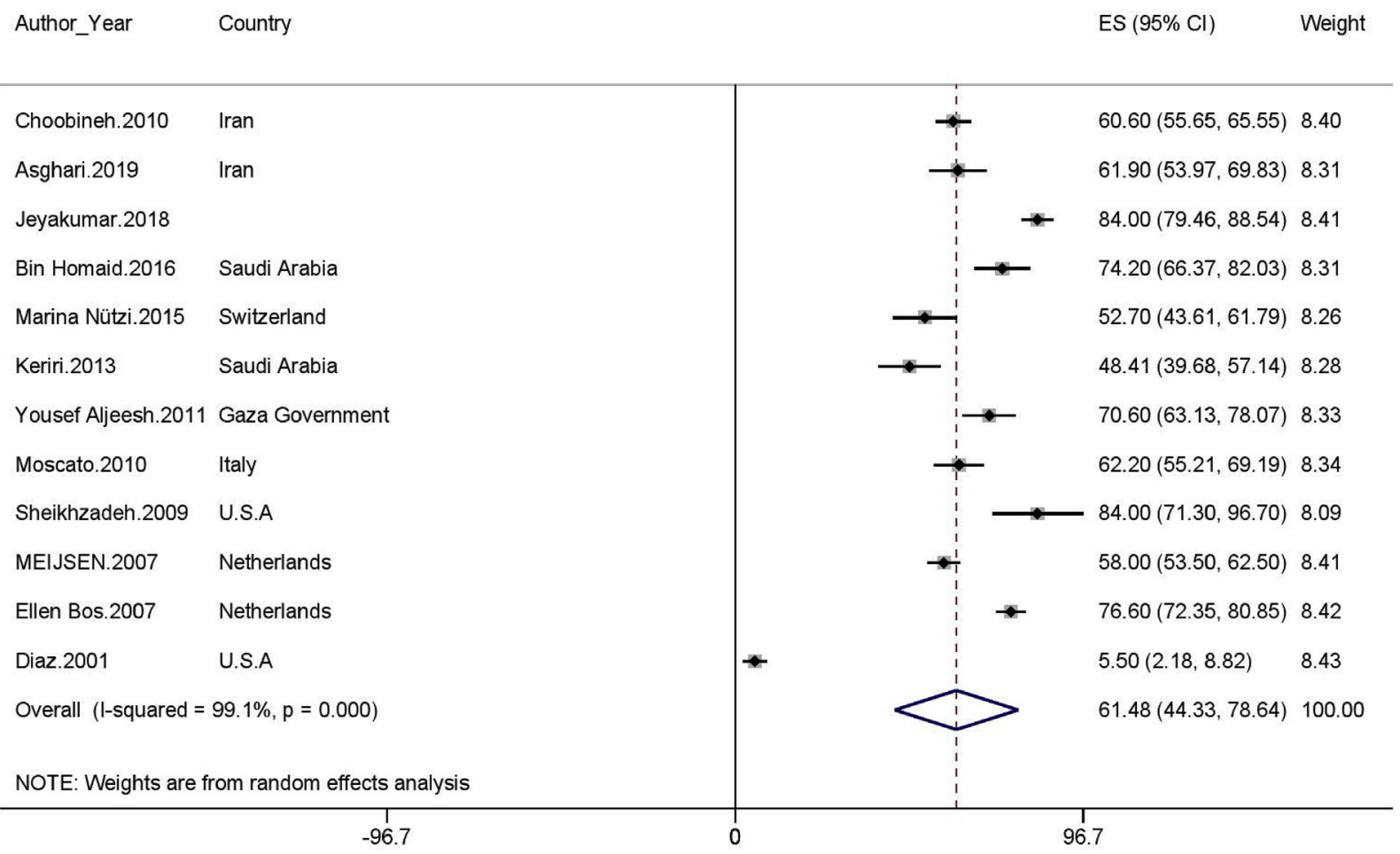

Fig. 7. Prevalence of musculoskeletal disorders in lower back amongst studies included. 


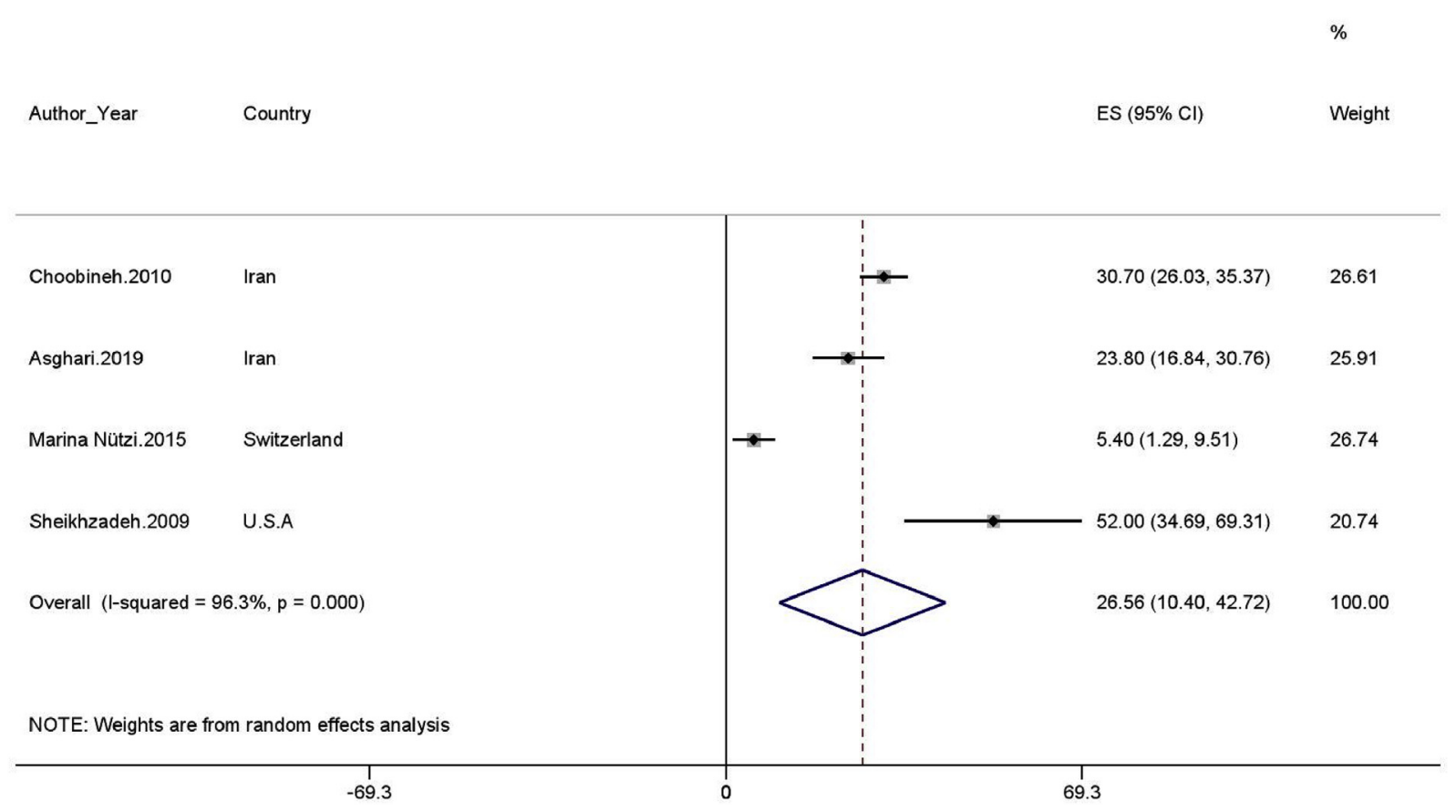

Fig. 8. Prevalence of musculoskeletal disorders in hip amongst studies included.

ES $(95 \% \mathrm{Cl}) \quad$ Weight

\begin{tabular}{|c|c|c|c|c|}
\hline Choobineh.2010 & Iran & & $58.10(53.11,63.09)$ & 20.64 \\
\hline Asghari.2019 & Iran & & $60.50(52.52,68.48)$ & 20.22 \\
\hline Marina Nützi.2015 & Switzerland & $\longrightarrow$ & $20.50(13.15,27.85)$ & 20.32 \\
\hline Sheikhzadeh.2009 & U.S.A & & $58.00(40.90,75.10)$ & 18.05 \\
\hline MEIJSEN.2007 & Netherlands & $\rightarrow$ & $22.00(18.23,25.77)$ & 20.76 \\
\hline \multicolumn{2}{|c|}{ Overall (I-squared $=97.9 \%, p=0.000)$} & & $43.43(23.80,63.06)$ & 100.00 \\
\hline \multicolumn{2}{|c|}{ NOTE: Weights are from random effects analysis } & & & \\
\hline
\end{tabular}

Fig. 9. Prevalence of musculoskeletal disorders in knees amongst studies included.

significant. The results also revealed no significant correlation between the personnel's age and the prevalence of MSDs (Table 2).

\subsection{Publication bias}

Finally, we created funnel plot to explore the possibility of publication bias, yet the results of Egger's test were no evident of this bias $(\mathrm{p}=0.458)$ (Fig. 11).

\section{Discussion}

The present systematic review and meta-analysis aimed to investigate the prevalence of MSDs among operating room personnel. After exploration of 12 articles, the results indicated that the highest prevalence of MSDs was related to lower back followed by ankles \& feet and shoulders, while the lowest prevalence was related to elbow and hip pain. Almost half of the included studies had made use of researcher-made questionnaires for estimating the prevalence of MSDs, while Nordic questionnaire was only employed in three investigations. The results revealed that the highest prevalence of MSDs was related to lower back (61.48\%) among the operating room personnel. This rate was found to be similar in Asian (63.14\%) and European (62.37\%) countries. In this context, various studies have shown that due to its high prevalence, back pain is one of the main reasons for absence from 


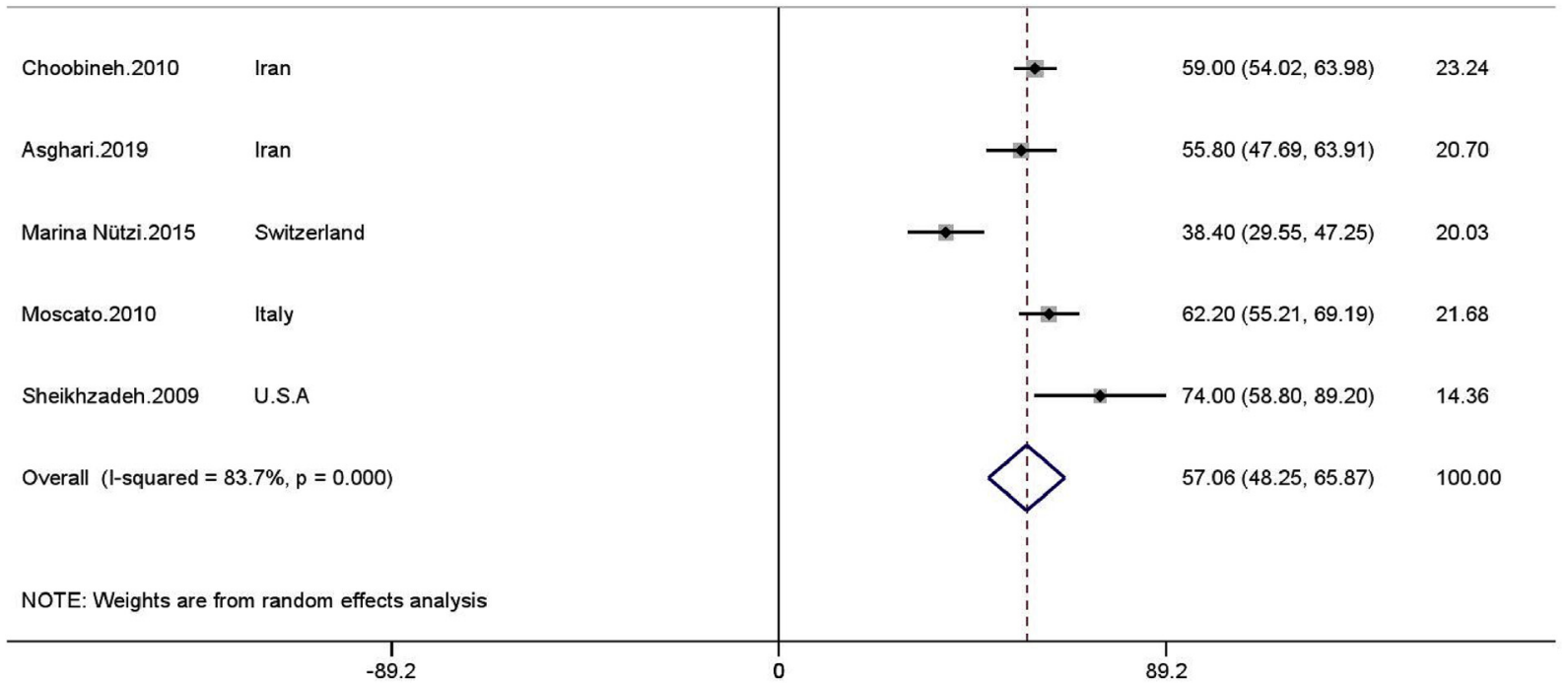

Fig. 10. Prevalence of musculoskeletal disorders in ankles $\&$ feet amongst studies included.

Table 2

Result of meta-regression of year of study and mean of age on musculoskeletal disorders among studies included.

\begin{tabular}{lllll}
\hline Organ/variable & \multicolumn{2}{l}{ Year of study } & \multicolumn{3}{l}{ Mean of age } \\
\cline { 2 - 5 } & Coefficient & P-value & Coefficient & P-value \\
\hline Neck & -0.032 & 0.432 & 0.029 & 0.382 \\
Shoulder & -0.052 & 0.080 & 0.045 & 0.290 \\
Elbow & -0.012 & 0.877 & 0.073 & 0.272 \\
Wrist \& hand & -0.081 & 0.295 & -0.008 & 0.937 \\
Upper back & -0.050 & 0.228 & -0.006 & 0.903 \\
Lower back & 0.067 & 0.075 & -0.009 & 0.887 \\
Hip & -0.096 & 0.500 & 0.004 & 0.974 \\
Knee & 0.023 & 0.740 & -0.029 & 0.686 \\
Ankle \& Feet & -0.029 & 0.334 & 0.004 & 0.883 \\
\hline
\end{tabular}

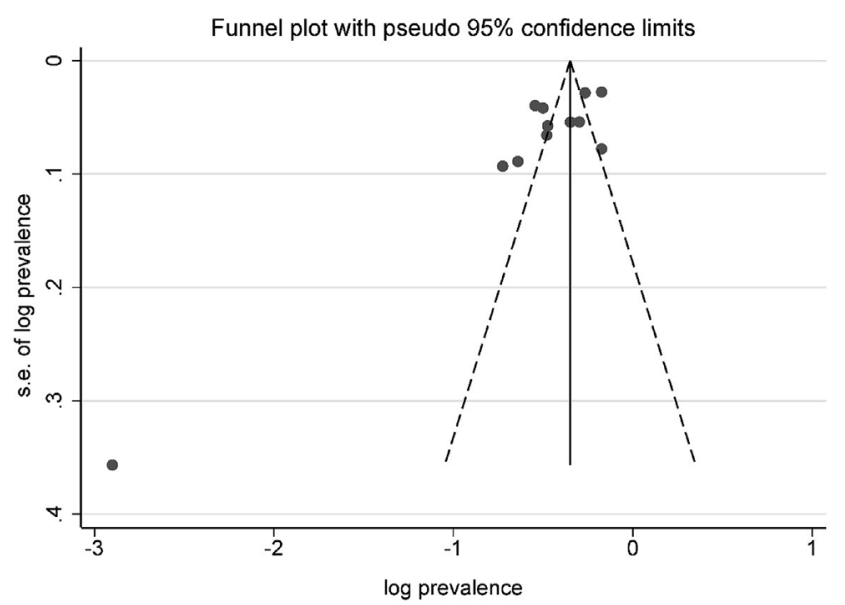

Fig. 11. Funnel plot to explore the possibility of publication bias.

work and even career change among the operating room personnel ${ }^{35,} \cdot{ }^{36}$ This disorder may result from long-term standing, fixed body posture, and force exertion under improper conditions, ${ }^{22}$ which are unavoidable in this occupation.
Up to now, numerous studies have been conducted on MSDs among hospital personnel, and the results have attributed these disorders to high physical demands. ${ }^{37-39}$ Nonetheless, limited interventions including training and mechanical aids have been considered for reducing physical demands and MSDs. A systematic review performed in 1997 indicated that other individual and occupational risk factors could play a role in the incidence of MSDs among operating room personnel. ${ }^{40}$ Other effective factors in these disorders included work-related psychosocial factors like boring work, occupational stress, lack of support, time pressure, job demands, job satisfaction, and staff insufficiency. In line with the present study findings, Long et al. stated that monotonous work, high job demands, and low job control could increase the risk of MSDs symptoms. ${ }^{41}$ Based on the biopsychosocial models concerning the impact of psychosocial factors on MSDs, psychosocial stressors in the workplace cause MSDs via creating physiological responses such as increased muscle tension. ${ }^{42}$ The psychological status resulting from occupational stressors leads to a physical reaction, which may manifest through health problems over time. ${ }^{43}$ Considering the extent of effective psychosocial factors in MSDs, these factors are recommended to be explored in future studies.

The current study findings revealed the highest prevalence of MSDs in ankles \& feet $(57.06 \%)$ after the back. The CCOHS has announced that continuous work in standing position can lead to leg pain, leg swelling, varicose veins, decreased muscle perfusion, general muscular fatigue, back pain, neck and shoulder stiffness, and other health problems. ${ }^{21}$ This disorder and its high prevalence can be justified by the operating room personnel's body posture (normally standing during work) as well as the studies conducted on the issue.

\subsection{Study limitations}

The present study had some limitations, one of the most important of which being the use of different questionnaires for investigation of MSDs. Therefore, not all organs were assessed in all studies and only low back was evaluated comprehensively. Hence, further studies are suggested to make use of a standard questionnaire, such as Nordic Musculoskeletal Questionnaire, for comprehensive collection of MSDs, so as to determine the results of these disorders in all body organs. 


\subsection{Recommendation for future research}

The present study recommend that future studies should review the physical and psychosocial factors affecting musculoskeletal disorders. Future studies can also compare the prevalence of musculoskeletal disorders in operating room staff with other medical personnel such as nurses and surgeons.

\section{Conclusion}

The study results revealed the high prevalence of MSDs, specifically in lower back, upper back, neck, shoulders, ankles, and feet, among the operating room personnel. Since the operating room is the most sensitive ward of any hospital and is called the heart of the hospital, due attention should be paid to the operating room personnel, because any disorder or challenge not only hurts the personnel, but it also exerts detrimental effects on patients and the healthcare system.

\section{Declaration of competing interest}

The authors declare no potential conflict of interest with respect to the research, authorship, and/or publication of this article.

\section{Acknowledgment}

We would like to acknowledge the support of Shiraz University of Medical Sciences for providing the funds for this research.

\section{References}

1. Sadeghian F, Hosseinzadeh S, Aliyari R. Do psychological factors increase the risk for low back pain among nurses? A comparing according to cross-sectional and prospective analysis. Saf Health Work. 2014;5(1):13-16.

2. Soroush A, Shamsi M, Izadi N, Heydarpour B, Samadzadeh S, Shahmohammadi A. Musculoskeletal disorders as common problems among iranian nurses: a systematic review and meta-analysis study. Int J Prev Med. 2018;9.

3. Anyfantis ID, Biska A. Musculoskeletal disorders among Greek physiotherapists: traditional and emerging risk factors. Saf Health Work. 2018;9(3):314-318.

4. Vos T, Flaxman AD, Naghavi M, et al. Years lived with disability (YLDs) for 1160 sequelae of 289 diseases and injuries 1990-2010: a systematic analysis for the Global Burden of Disease Study 2010. Lancet. 2012;380(9859):2163-2196.

5. Niu S. Ergonomics and occupational safety and health: an ILO perspective. Appl Ergon. 2010;41(6):744-753.

6. Park J, Kim Y, Han B. Work sectors with high risk for work-related musculoskeletal disorders in Korean men and women. Saf Health Work. 2018;9(1):75-78.

7. Nuraydın A, Ö Bilek, Kenziman AK, et al. The mersin greenhouse workers study. Surveillance of work-related skin, respiratory, and musculoskeletal diseases. Ann Global Health. 2018;84(3):504-511

8. Punnett L, Wegman DH. Work-related musculoskeletal disorders: the epidemiologic evidence and the debate. J Electromyogr Kinesiol. 2004;14(1):13-23.

9. Davey S, Bulat E, Massawe H, Pallangyo A, Premkumar A, Sheth N. The economic burden of non-fatal musculoskeletal injuries in Northeastern Tanzania. Ann Global Health. 2019;85(1).

10. Bernard BP, Putz-Anderson V. Musculoskeletal Disorders and Workplace Factors; a Critical Review of Epidemiologic Evidence for Work-Related Musculoskeletal Disorders of the Neck, Upper Extremity, and Low Back. 1997; 1997.

11. Labao HC, Faller EM, Bacayo MFD. 'Aches and Pains' of Filipino migrant workers in Malaysia: a profile of work-related musculoskeletal disorders. Ann Global Health. 2018;84(3):474-480.

12. Richardson A, McNoe B, Derrett S, Harcombe H. Interventions to prevent and reduce the impact of musculoskeletal injuries among nurses: a systematic review. Int $J$ Nurs Stud. 2018;82:58-67.

13. Saberipour B, Ghanbari S, Zarea K, Gheibizadeh M, Zahedian M. Investigating prevalence of musculoskeletal disorders among Iranian nurses: a systematic review and meta-analysis. Clin Epidemiol Global Health. 2019;7(3):513-518.

14. Shafiezadeh K. Prevalence of musculoskeletal disorders among paramedics working in a large hospital in Ahwaz, southwestern Iran in 2010. Int J Occup Environ Med.
2011

15. Hoboubi N, Choobineh A, Ghanavati FK, et al. Relationship between organizational leadership style and musculoskeletal injuries among workers of an Iranian process industry. Shiraz E Med J. 2018;19(11)

16. Abedini R, Choobineh A, Hasanzadeh J. Musculoskeletal load assessment in hospital nurses with patient transfer activity. Int J Occup Hyg. 2013;5(2):39-45.

17. Choobineh A, Rajaeefard A, Neghab M. Association between perceived demands and musculoskeletal disorders among hospital nurses of Shiraz University of Medical Sciences: a questionnaire survey. Int J Occup Saf Ergon. 2006;12(4):409-416.

18. Afra A, Mollaei Pardeh M, Saki H, et al. Anesthetic Toxic Isoflurane and Health Risk Assessment in the Operation Room in Abadan, Iran during 2018. Clinical Epidemiology and Global Health; 2019.

19. Attar SM. Frequency and risk factors of musculoskeletal pain in nurses at a tertiary centre in Jeddah, Saudi Arabia: a cross sectional study. BMC Res Notes. 2014;7(1):61.

20. Andersen JH, Haahr JP, Frost P. Risk factors for more severe regional musculoskeletal symptoms: a two-year prospective study of a general working population. Arthritis Rheum. 2007;56(4):1355-1364.

21. Bank HSD. Canadian centre for occupational health and safety. Far E Q. 1998;1.

22. Choobineh A, Movahed M, Tabatabaie SH, Kumashiro M. Perceived demands and musculoskeletal disorders in operating room nurses of Shiraz city hospitals. Ind Health. 2010;48(1):74-84.

23. Karimian R, Rahnama N, Habibi E, Ghasemi GA, Karimian M. The effect OF corrective exercises ON musculoskeletal disorders. Health Syst Res. 2010;6(3)

24. Smith DR, Mihashi M, Adachi Y, Koga H, Ishitake T. A detailed analysis of musculoskeletal disorder risk factors among Japanese nurses. J Saf Res. 2006;37(2):195-200.

25. Nützi M, Koch P, Baur H, Elfering A. Work-Family conflict, task interruptions, and influence at work predict musculoskeletal pain in operating room nurses. Saf Health Work. 2015;6(4):329-337.

26. Asghari E, Dianat I, Abdollahzadeh F, et al. Musculoskeletal pain in operating roon nurses: associations with quality of work life, working posture, socio-demographic and job characteristics. Int J Ind Ergon. 2019;72:330-337.

27. Homaid MB, Abdelmoety D, Alshareef W, et al. Prevalence and risk factors of low back pain among operation room staff at a Tertiary Care Center, Makkah, Saudi Arabia: a cross-sectional study. Ann Occup Environ Med. 2016;28(1):1.

28. Keriri H. Prevalence and risk factors of low back pain among nurses in operating rooms, Taif, Saudi Arabia. Am J Res Commun. 2013;1(11):25.

29. Diaz JH. Carpal tunnel syndrome in female nurse anesthetists versus operating room nurses: prevalence, laterality, and impact of handedness. Anesth Analg. 2001;93(4):975-980

30. Sheikhzadeh A, Gore C, Zuckerman JD, Nordin M. Perioperating nurses and technicians' perceptions of ergonomic risk factors in the surgical environment. Appl Ergon. 2009;40(5):833-839.

31. Bos E, Krol B, van der Star L, Groothoff J. Risk factors and musculoskeletal complaints in non-specialized nurses, IC nurses, operation room nurses, and X-ray technologists. Int Arch Occup Environ Health. 2007;80(3):198-206.

32. Meijsen P, Knibbe HJ. Work-related musculoskeletal disorders of perioperative personnel in The Netherlands. AORN J. 2007;86(2):193-208

33. Moscato U, Trinca D, Rega ML, et al. Musculoskeletal injuries among operating room nurses: results from a multicenter survey in Rome, Italy. $J$ Public Health. 2010;18(5):453-459.

34. Aljeesh YI, Nawajha S. Determinants of low back pain among operating room nurses in gaza governmental hospitals. Determinants of low back pain among operating room nurses in gaza governmental hospitals. 2011; 2011:14.

35. Bhandary AK, Chimes GP, Malanga GA. Investigational pharmacology for low back pain. J Pain Res. 2010;3:169.

36. Phillips N. Berry \& Kohn's Operating Room Technique. Elsevier Health Sciences; 2016

37. Alexopoulos EC, Burdorf A, Kalokerinou A. Risk factors for musculoskeletal disorder among nursing personnel in Greek hospitals. Int Arch Occup Environ Health. 2003;76(4):289-294

38. Harcombe H, McBride D, Derrett S, Gray A. Prevalence and impact of musculoskeletal disorders in New Zealand nurses, postal workers and office workers. Aust $N \mathrm{ZJ}$ Publ Health. 2009;33(5):437-441.

39. Smith DR, Wei N, Zhao L, Wang R-S. Musculoskeletal complaints and psychosocial risk factors among Chinese hospital nurses. Occup Med. 2004:54(8):579-582.

40. Bernal D, Campos-Serna J, Tobias A, Vargas-Prada S, Benavides FG, Serra C. Workrelated psychosocial risk factors and musculoskeletal disorders in hospital nurses and nursing aides: a systematic review and meta-analysis. Int $J$ Nurs Stud. 2015;52(2):635-648.

41. Lang J, Ochsmann E, Kraus T, Lang JW. Psychosocial work stressors as antecedents of musculoskeletal problems: a systematic review and meta-analysis of stability-adjusted longitudinal studies. Soc Sci Med. 2012;75(7):1163-1174.

42. Melin B, Lundberg U. A biopsychosocial approach to work-stress and musculosketal disorders. J Psychophysiol. 1997.

43. McEwen BS. Protective and damaging effects of stress mediators. $N$ Engl $J$ Med. 1998;338(3):171-179. 